\title{
Cell-free fetal DNA and non-invasive prenatal diagnosis
}

Currently in the UK, prenatal diagnosis of genetic conditions and Down's syndrome requires invasive diagnostic tests such as amniocentesis and chorionic villus sampling (CVS). Procedural related miscarriage rates of about $1 \%$ have been quoted for these tests which are not usually done before 11 weeks' gestation. ${ }^{1}$ Annually in the UK, 32000 women have an invasive diagnostic test as a result of other screening tests, indicating that they are at increased risk for their children having Down's syndrome. Around a further 1500 pregnant women have an invasive test because the fetus is at high risk of a genetic condition.

An area that has generated much interest in recent years has been in isolating fetal genetic material present in maternal blood as a target for non-invasive prenatal diagnosis (NIPD). In 1997 Lo et a/ ${ }^{2}$ identified cell-free fetal DNA in the maternal circulation. This finding has generated research in the development of clinical applications based on analysing this fetal genetic material for NIPD. Cell-free fetal DNA represents extracellular DNA which originates from trophoblastic cells. ${ }^{3}$ However, the vast majority of cell-free DNA in maternal blood originates from the mother, with cell-free fetal DNA representing only $3 \%$ of the total cell-free circulating DNA in early pregnancy rising to $6 \%$ in late pregnancy. ${ }^{4}$

After delivery, cell-free fetal DNA is rapidly cleared from the maternal circulation, making it specific to that pregnancy. As the fetal DNA is swamped by the presence of cell-free maternal DNA, the challenge has been to separate the fetal from the maternal cell-free DNA. Various methods have been used for this, including using methods based on the fact that the fetal DNA is shorter than maternal DNA. However, it is still not possible to extract pure fetal DNA and so currently prenatal diagnosis using cell-free fetal DNA is limited to the detection or exclusion of genetic sequences that are not present in the mother, that is, determination of fetal sex, fetal rhesus D status in D-negative mothers, or genetic conditions inherited from the father or arising de novo. ${ }^{5}$

The other approach to NIPD that is being explored is the analysis of fetal RNA in the maternal circulation. Researchers have identified genes that are expressed in the placenta, but not the mother, and are therefore specific to the fetus. ${ }^{6}$ Subsequent identification of messenger RNA from these genes in maternal circulation must therefore be fetal specific.

The Special Non-Invasive Advances in Fetal and Neonatal Evaluation (SAFE) Network of Excellence, a European Commission funded network of laboratories and clinicians, has worked to overcome the technical challenges and identify the limitations in the use of the current technology. ${ }^{7}$ The group has also addressed many of the social, ethical, and economic challenges. This network will end in 2009, but there are two groups in the UK funded to investigate the routine implementation of fetal rhesus-D typing, and other applications in clinical practice, and so evaluate NIPD thoroughly including the sensitivity, specificity, and clinical utility with development of laboratory and clinical standards before it 'seeps' further into practice.

Rapid progress is being made in this area, and the challenge for primary care teams is keeping up to date with current advances and to develop the necessary competencies to be able to advise women and couples. This editorial outlines the status of current research and the clinical applications of NIPD, highlighting areas which may come into practice in the near future as this is a very rapidly developing area.

\section{RHESUS-D STATUS}

Clinical studies have already demonstrated the potential benefit and impact of using cell-free fetal DNA in NIPD. The presence of rhesus $D$ gene sequences in analysing fetal free DNA in D-negative women indicates that the fetus is D-positive; if the rhesus $D$ sequence is not present then the fetus is predicted to be D-negative. Many supportive studies have demonstrated its diagnostic sensitivity which ranges from $95-100 \%$ with specificities of over $99 \% .^{8}$ The analysis of cell-free fetal DNA is now the method of choice in the management of pregnancies at high risk of haemolytic disease of the newborn. ${ }^{8}$ For several years now the management of mothers with a history of haemolytic disease of the newborn or antibodies to rhesus $D$ has involved non-invasive prenatal diagnosis to determine the fetal rhesus $D$ status. If the fetus is predicted to be D-negative no further monitoring is required, but if it is Dpositive close monitoring in a fetal medicine unit is needed.

These tests have been done using labour intensive laboratory methods but, if high throughput technology can be developed, there is potential for use routinely. Consider that in the UK anti-D prophylaxis is given to all D-negative women usually in the third trimester, and after feto-maternal haemorrhage as well as after birth if the baby is D-positive. However, around $40 \%$ of D-negative women carry a D-negative fetus and so do not require anti-D prophylaxis. Anti-D is a human blood product and thus reducing its use in women who do not need it will reduce potential exposure to hepatitis $\mathrm{C}$ and prion type diseases $^{9}$ as well as NHS expenditure. A high throughput method for reliable analysis of fetal rhesus $D$ status at 28 weeks has been described recently ${ }^{10}$ and the challenge now is to refine the technology for routine use earlier in pregnancy and thus avoid unnecessary administration of anti-D.

\section{FETAL SEX DETERMINATION}

One other current clinical application is fetal sex determination which involves identifying Y-chromosome genetic markers in maternal blood. This has been shown to be accurate from around 7 weeks' gestation ${ }^{11}$ and a recent audit of cases done in the UK gave an accuracy of $97 \%$ when done at 7 weeks or later. This test can be offered to women at risk of X-linked genetic conditions such as Duchenne muscular 
dystrophy. In this situation, only women carrying male fetuses require an invasive test to determine whether or not the fetus is thus affected.

Fetal sex determination can also aid diagnosis where there is genital ambiguity on ultrasound and in the management of metabolic conditions, such as congenital adrenal hyperplasia. Knowledge of the fetal sex at an early stage allows for early cessation of this drug. However, the use of this early and safer method for fetal sex determination illustrates the potential ethical and social aspects of using the technology. This technology is already offered via the internet for home testing in the US and the ready availability leaves scope for further discussion regarding access to genetic testing from commercial sources, rather than through health professionals. $^{12}$

\section{SINGLE GENE DISORDERS}

NIPD for single gene disorders, such as achondroplasia or Huntingdon's disease, requires the identification of modified genes in maternal circulation and can currently only be done to detect a gene change that is not present in the mother. This can only be used to diagnose or exclude genes inherited from the father or those arising de novo. At present there are only a few reports of NIPD for this purpose in the literature, ${ }^{4}$ but it is hoped that over the next few years prenatal diagnosis for these families at high risk of genetic conditions will be available earlier in pregnancy, and be safer.

\section{NIPD FOR DOWN'S SYNDROME}

NIPD for Down's syndrome poses different challenges. At the moment, while we are still unable to separate free fetal DNA completely from the cell-free fetal DNA, identification of the very small amount of extra fetal chromosome 21 is virtually impossible. However, using the mRNA from a gene, PLAC4, which is expressed only in the placenta, Lo et al have correctly identified nine of 10 Down's syndrome fetuses. ${ }^{13}$ They have also successfully used methods that could be used in routine clinical practice. ${ }^{14}$ Another group reported using the new genetic sequencers, which can detect very small changes in the quantity of different DNA sequences, for accurate diagnosis of Down's, Edwards', and Patau's syndromes. ${ }^{15}$ As each human chromosome has a different pattern of sequences, the different chromosomes can be distinguished using this methodology and the relative quantity of a chromosome determined. If a fetus has Down's syndrome there will be a relative increase in the quantity of chromosome 21 in the mother's blood, the fetus having three copies of 21 rather than two. Although there is relatively little cell-free fetal DNA in mother's blood, the majority coming from her, these sequencers are sensitive enough to detect this very small increase in amount of chromosome 21.

A lot more work needs to be done before these tests can be used to replace diagnostic CVS or amniocentesis, both to determine the sensitivity and specificity of these tests, but also to develop the infrastructure required for implementation in terms of education, service delivery, and laboratory facilities. A large study will start soon in the US and UK to see whether these techniques can be useful in high-risk women, or, possibly to replace the current Down's syndrome screening tests.

\section{SUMMARY}

The potential for change in the delivery of prenatal diagnosis and screening secondary to these new technologies is vast. The likelihood is that there will be a significant change in how we counsel women and couples who are considering prenatal screening and genetic diagnosis. This will demand that primary care teams have the necessary competencies to advise women and couples. There are many technical and ethical hurdles to overcome. We will need to maintain high standards of counselling to facilitate informed consent taking account of cultural variation. ${ }^{16}$ Of prime importance will be both healthcare professional and public education.

The message for primary care is clear. The technology exists for novel approaches to prenatal diagnosis. There are already clinical applications. Primary care teams need to be aware of the potential for earlier and safer prenatal diagnosis to facilitate timely and appropriate referral to genetic or obstetric teams. In particular, those offering community-based antenatal care need to be aware of the developments in Down's syndrome diagnosis and screening, albeit for the time being to be in a position to inform women and their families that these technologies are still in the developmental phase. There is no doubt that an understanding of the science with the ability to communicate this to the public will be key for the effective delivery of this exciting breakthrough in fetal medicine and antenatal care. This communication must start in the community.

\section{Imran Rafi,}

Senior Lecturer in Primary Care Education. Department of Community Health Sciences, St George's University of London.

\section{Lyn Chitty,}

Reader in Genetics and Fetal Medicine, Clinical and Molecular Genetics Unit, Institute of Child Health and Fetal Medicine Unit, University College London Hospitals NHS Foundation Trust.

\section{Acknowledgements}

LSC is partly supported by Biomedical Research Centre funding from the Department of Health. She is a member of the Special Non-Invasive Advances in Fetal and Neonatal Evaluation (SAFE) Network of Excellence (LSH-CT-2004-503241) and acknowledges the support from the European Commission, who funded this network.

\section{REFERENCES}

1. Mujezinovic F, Alfirevic Z. Procedure-related complications of amniocentesis and chorionic villous sampling: a systematic review. Obstet Gynecol 2007; 110(3): 687-694.

2. Lo YM, Corbetta N, Chamberlain PF, et al. Presence of fetal DNA in maternal plasma and serum. Lancet 1997; 350(9560): 485-487.

3. Alberry M, Maddocks D, Jones M, et al. Free fetal DNA in maternal plasma in anembryonic pregnancies: confirmation that the origin is the trophoblast. Prenat Diagn 2007; 27(5): 415-418.

4. Lo YM, Tein MS, Lau TK. Quantitative analysis of fetal DNA in maternal plasma and serum: implications for noninvasive prenatal diagnosis. Am J Hum Genet 1998; 62(4): 768-775.

5. Hahn S, Chitty LS. Noninvasive prenatal diagnosis: current practice and future perspectives. Curr Opin Obstet Gynecol 2008; 20(2): 146-151.

6. Ng EK, Tsui NB, Lau TK et al. mRNA of placental origin is readily detectable in maternal plasma. Proc Natl Acad Sci USA 2003; 100(8): 4748-4753

7. Chitty LS, van der Schoot CE, Hahn S, Avent ND. SAFE-The Special Non-invasive Advances in Fetal and Neonatal Evaluation Network: aims and achievements. Prenat Diagn 2008; 28(2): 83-88.

8. Van der Schoot CE, Hayn S and Chitty LS. Non-invasive prenatal diagnosis and determinism of fetal Rh status. Semin Fetal Neonatal Med 2008; 13(2): 63-68.

9. Kenny-Walsh E. Clinical outcomes after hepatitis C infection from contaminated anti-D immune globulin. Irish Hepatology Research Group. N Engl J Med 1999; 340(16): 1228-1233.

10. Finning K, Martin P, Summers J, et al. Effect of high throughput RHD typing of fetal DNA in maternal plasma on use of anti-RhD immunoglobulin in $\mathrm{RhD}$ negative pregnant women: prospective feasibility study. BMJ 2008; 336(7648): 816-818.

11. Hyett JA, Gardiner G, Stojilkovic-Mikic T, et al. 
Reduction in diagnostic and therapeutic interventions by non-invasive determination of fetal sex in early pregnancy. Prenat Diagn 2005; 25(12): 1111-1116.

12. Newson AJ. Ethical aspects arising from non-invasive fetal diagnosis. Semin Fetal Neonatal Med 2008; 13(2): 103-108.

13. Lo YM, Tsui NB, Chiu RW, et al. Plasma placental RNA allelic ratio permits noninvasive prenatal chromosomal aneuploidy detection. Nat Med 2007; 13(2): 218-223.

14. Lo YM, Lun FM, Chan KC, et al. Digital PCR for the molecular detection of fetal chromosomal aneuploidy.
Proc Natl Acad Sci USA 2007; 104(32): 13116-13121.

15. Fan HC, Blemenfeld YJ, Hudgins L et al. Noninvasive diagnosis of fetal aneuploidy by shotgun sequencing DNA from maternal blood. Proc Nat Acad Sci USA 2008; 105(42): 16266-16271.

16. Van den Heuvel A, Marteau TM. Cultural variation in values attached to informed choice in the context of prenatal diagnosis. Semin Fetal Neonatal Med 2008: 13(2): 99-102.

DOI: 10.3399/bjgp09X420572
ADDRESS FOR CORRESPONDENCE

\section{Imran Rafi}

Department of Community Health Sciences, Hunter Wing, St George's University of

London, Cranmer Terrace, Blackshaw Road, Tooting, London, SW17 ORE.

E-mail: irafi@sgul.ac.uk 